\title{
Handwritten Kannada Numeral Recognition using Radial Basis Function
}

\author{
Shivanand Rumma \\ Chairman \\ Dept. of P.G. Studies in \\ Computer Science \\ Govt. First grade College, Bidar
}

\author{
Vishweshwarayya C. H. \\ Dept. of MCA \\ Angadi Institute of Technology \\ \& Management, Belgaum
}

\author{
Bhuvaneshwari B. D. \\ Dept. of Comp. Science \\ Karnataka University Darawad,
}

\begin{abstract}
It is great importance of recognizing handwritten numerals using a classifier. This paper applies the technique of Radial Basis Function for handwritten numeral recognition of Kannada script. Much work has been done on Kannada numeral recognition using different techniques for getting the more accuracy of recognition. Since different researchers have different techniques, so here we are trying to form a system where recognition of numerals becomes easy. An algorithm is proposed for determining Kannada numeral recognition using above mentioned system.
\end{abstract}

\section{Keywords}

Radial basis function(RBF),Kannada Numeral Recognition, K-means clustering, Principal Component Analysis(PCA).

\section{INTRODUCTION}

The industrial application of character and document recognition in the last 40 years have been in for reading, bank cheque reading and postal address reading. From these applications recognition capability has expanded in multiple dimensions: mode of writing, scripts, types of documents and so on. The different types of recognizable modes of writing are machine printing, hand printing and script hand writing.

The numeral recognition field has not always been peaceful. It has twice been disturbed by trends of new digital technologies that threatened to make less role of OCR technology. The first such trend was that of office automation in 1980's. Most of information seemed to be going to be born digital, potentially becoming less demand for OCR's and some researchers were pessimistic about the future

Now we are facing the second trend which is IT and Web technologies. Many kinds of applications can now be completed on the web .Information can flow around the world within fractions of second. However it is still not known whether the demand for numeral recognition will decrease or whether new applications requiring more advanced technology will be created.

Handwritten recognition[1] is the ability of a computer to receive and interpret handwritten numeral input from sources such as document, photographs, touch screens and other devices. In past, the algorithms are proposed in numerals of different languages like English[2],Chinese[3], Arabian[4], Persian, Bengali, Gujarati etc. In neural network different classifiers have been used for handwritten digit recognition.

Here we proposed the method for recognition of handwritten Kannada numerals using Radial Basis Function in this paper. It is feed forward neural network that computes the activation at the hidden neuron in a way that is different from input vector and the weight vector. Hidden neuron activation in
$\mathrm{RBF}$ are computed using an exponential of distance measure between the input vector and the prototype vector.

This paper is organized in the following manner, section 2 gives information about radial basis function, system overview is explained in section 3 and results and conclusions are explained in section 4 .

\section{กง}

Fig 1:Kannada numerals

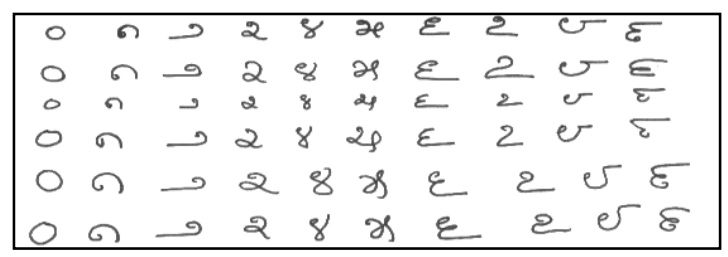

Fig 2 : Handwritten Kannada numerals

\section{RADIAL BASIS FUNCTION (RBF)}

$\mathrm{RBF}$ was originally introduced into the literature for the purpose of interpolation of data points on known number of training data sets[4].The three layers in this network are input layers, hidden layers with non linear RBF[5] activation function and linear output layer. Hidden layer performs mapping from the input space into higher dimensional space in which the patterns become linearly separable. The network output $\varphi$ for $\mathrm{N}$ number of neurons in the hidden layer is

$$
\varphi(x)=\sum a_{i} \sigma\left(\left\|x-C_{i}\right\|\right)
$$

Where $C_{i}$ is the center vector of neurons, $i$ and $a_{i}$ are the weight of the linear output neurons.

\section{SYSTEM OVERVIEW}

The recognition of Kannada handwritten numerals can be broadly classified into three blocks :
1. Pre-processing
2. Feature Extraction
3. Radial Basis Function Classifier. 


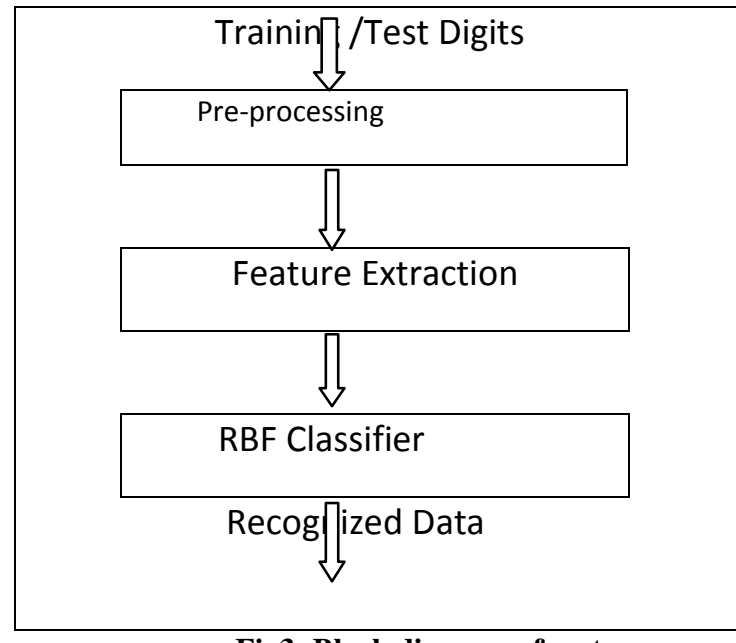

Fig3: Block diagram of system

\subsection{Pre-Processing}

Here objective is to obtain a set of hand written samples of Kannada numerals. There is a variation in style of writing from person to person. Noise and distortion present in the input text due to hardware and software limitations and also size of numerals varies from person to person .Hence size normalization and noise removal are done i pre-processing step. In size normalization, the input image is segmented into characters. These segmented characters are resize to size of $36 * 36$ pixels.

Noise in the document may be due to poor quality of papers, or due to dust in paper while scanning the document, such noise can be removed using filters.

$$
\begin{aligned}
& \circ \cap \rightarrow 2 \& \text { \& } 2 \text { ए ह } \\
& \circ \curvearrowleft \rightarrow 2 \text { \& } \Rightarrow \varepsilon \varepsilon \varepsilon
\end{aligned}
$$

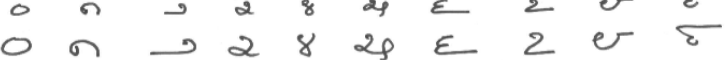

$$
\begin{aligned}
& 0 \Omega \rightarrow 28 \text { \& } 2 \text { ह } \varepsilon \\
& 0 \Omega 28 \text { \& } 2 \text { e ह }
\end{aligned}
$$

Fig 4: Preprocessed Handwritten Kannada Numerals

$$
\begin{aligned}
& 0 \text { ด } 2829 \varepsilon 2 v \varepsilon \\
& 0 \text { ด } 28 \text { भ E ऐ } v \\
& 0 \text { ด } 28 \partial \varepsilon 20 \varepsilon
\end{aligned}
$$

Fig 5: Numeral Image is Segmented into lines

\section{$8 x 2 ?$}

Fig 6: Line is segmented in to individual numeral

\subsection{Feature Extraction}

There are three types of feature extractions namely statistical, structural and hybrid features. Scale invariant feature transformation[6] is used to transfer the character image into a set of local features.

In statistical technique, pixel density is calculated for each zone. Then the pixel density is used for representing the features. The dimensions height and width of the character pixels are counted using encoding Binary variation approach.

In hybrid technique, Hough transform [7] is used to detect the horizontal and vertical lines.

\subsection{RBF Classifier}

Calculation of canters for the basis function and network is given in figure 3.

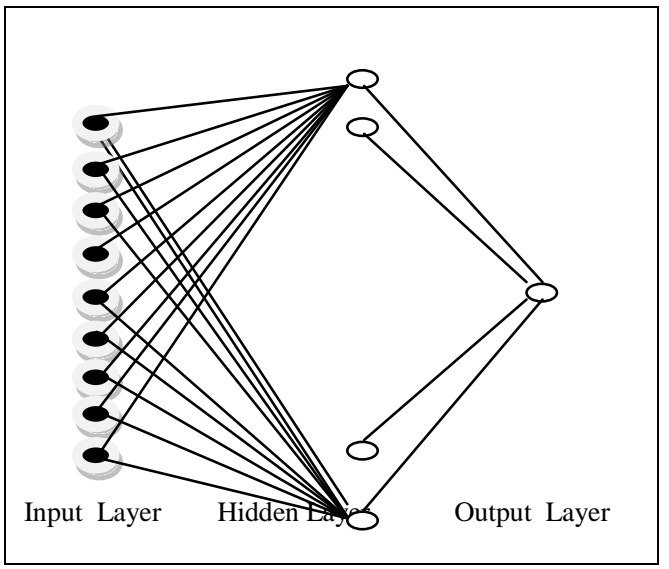

Fig7: Radial Basis Function Network

\section{Algorithm for Pattern Recognition Using RBF}

Input: Image of Kannada handwritten numeral from test set.

\section{Process}

1. Form the training set of patterns after normalizing the size and removal of noise.

2. Extract important feature for each data to be stored in cluster.

3. Using PCA remove the redundant data items.

\section{Output:}

1. Center of basis function is calculated.

2. Determine weights between the hidden neuron.

3. Input data matches with any of the cluster for the

\begin{tabular}{|c|c|c|c|c|c|c|c|c|c|c|}
\hline $\mathbf{I} / \mathbf{P}$ & C0 & $\mathbf{C 1}$ & C2 & C3 & $\mathrm{C4}$ & C5 & C6 & C7 & C8 & C9 \\
\hline 0 & 1 & 0 & 0 & 0 & 0 & 0 & 0 & 0 & 0 & 0 \\
\hline$\Omega$ & 0 & 1 & 0 & 0 & 0 & 0 & 0 & 0 & 0 & 0 \\
\hline פو & 0 & 0 & 1 & 0 & 0 & 0 & 0 & 0 & 0 & 0 \\
\hline 2 & 0 & 0 & 0 & 1 & 0 & 0 & 0 & 0 & 0 & 0 \\
\hline 8 & 0 & 0 & 0 & 0 & 1 & 0 & 0 & 0 & 0 & 0 \\
\hline $\mathscr{P}$ & 0 & 0 & 0 & 0 & 0 & 1 & 0 & 0 & 0 & 0 \\
\hline ᄅ & 0 & 0 & 0 & 0 & 0 & 0 & 1 & 0 & 0 & 0 \\
\hline 2 & 0 & 0 & 0 & 0 & 0 & 0 & 0 & 1 & 0 & 0 \\
\hline es & 0 & 0 & 0 & 0 & 0 & 0 & 0 & 0 & 1 & 0 \\
\hline$\sigma$ & 0 & 0 & 0 & 0 & 0 & 0 & 0 & 0 & 0 & 1 \\
\hline
\end{tabular}
particular cluster weight $=1$ otherwise 0 .

4. Once the values are calculated then the output can be determined by summing the values of each neuron.

\section{RESULT AND CONCLUSION}


$\mathrm{C}_{0} \ldots . . . \mathrm{C}_{9}$ represents the cluster having information regarding $0,1,2$ and so on upto 9 of Kannada numerals. If input is matched with data of the cluster then the cluster value will be 1 otherwise 0 .

In this proposed work, we tried to recognize Kannada numerals using RBF. It has advantages of easier to interpret the hidden layer in RBF.It has disadvantage that it is slower than a MLP.

In future, we would like to implement this proposed work using MATLAB.

\section{REFERENCES}

[1] "Handwritten Character Recognition using Neural Network" ,Sunith Bandaru.

[2] "Handwritten English Character Recognition Using Neural Networks", Anita Pal and Dayashanker Singh,vol 1,no 2, July-December 2010.
[3] "Printed Chinese Character Recognition", thesis by Yuan Lui.

[4] "Neural Networks", Simon Haykins.

[5] "Off-line handwritten characters recognition using Radial Basis Function”, J.Ashok and Dr.E.G.Rajan,vol 2,issue 04.

[6] Subashini A and Kodikara N.D, "A novel SIFE based codebook generation for handwritten Tamil character recognition" , $6^{\text {th }}$ IEEE Int. Conf. on Industrial and Information Systems (ICIIS), page(s):261-264, 2011

[7] Akshay Apte and Harshad Gado, "Tamil character recognition using structural features", 2010. 